\title{
Constrained and regularized system identification
}

\author{
TOR A. JOHANSEN† \\ Keywords: Regularization, Optimization, Parameter Estimation, Nonlinear \\ Systems
}

Prior knowledge can be introduced into system identification problems in terms of constraints on the parameter space, or regularizing penalty functions in a prediction error criterion. The contribution of this work is mainly an extension of the well known FPE (Final Production Error) statistic to the case when the system identification problem is constrained and contains a regularization penalty. The FPECR statistic (Final Production Error with Constraints and Regularization) is of potential interest as a criterion for selection of both regularization parameters and structural parameters such as order.

\section{Introduction}

In practical system identification it is often desirable to introduce prior knowledge into the problem, rather than relying completely on the data. If the model structure is assumed to be fixed, there are still several approaches, cf. Figure 1:

(1) Constraints on the parameter space, for example to ensure stability (Tulleken 1993, Johansen 1996a), convexity of an optimal control criterion (Foss and Johansen 1997), fulfillment of balance equations and steady-state data (Kramer et al. 1992, Thompson and Kramer 1994), frequency-domain data (Eskinat $e t$ al. 1993, Eskinat 1995), and explicit belief about parameter values (Bai and Sastry 1986). When the model is overparameterized, additional equality constraints are needed to make the problem well-posed and to avoid trivial solutions (Gawthrop et al. 1992, De Moor et al. 1994, Moons and De Moor 1995).

(2) Regularization, i.e. penalties on non-smooth behavior of the model (Tikhonov and Arsenin 1977, Larsen and Hansen 1994, Johansen 1997), deviation from explicit belief about parameters, and deviation from a prior known model (Kramer et al. 1992, Thompson and Kramer 1994, Johansen 1996a). Regularization is a general method that improves the robustness and allows identification of non-parsimonious models (Dayal and MacGregor 1996, Sjöberg et al. 1994, Sjöberg et al. 1993).

Prior knowledge in terms of constraints and penalties can be implemented directly in a prediction error method (PEM) framework (Johansen 1996a), or the penalties can be reformulated into equivalent prior distributions in a Bayesian system identification

Received 8 February 1998.

†Department of Engineering Cybernetics, Norwegian University of Science and Technology, N-7034 Trondheim, Norway. E-mail: Tor.Arne.Johansen@itk.ntnu.no

Reprinted from Preprints IFAC Symposium on System Identification SYSID '97, Kita Kyushu, Japan, pages 1467-1472, with permission from Elsevier Science Ltd., The Boulevard, Langford Lane, Kidlington OX5 1GB, UK. 


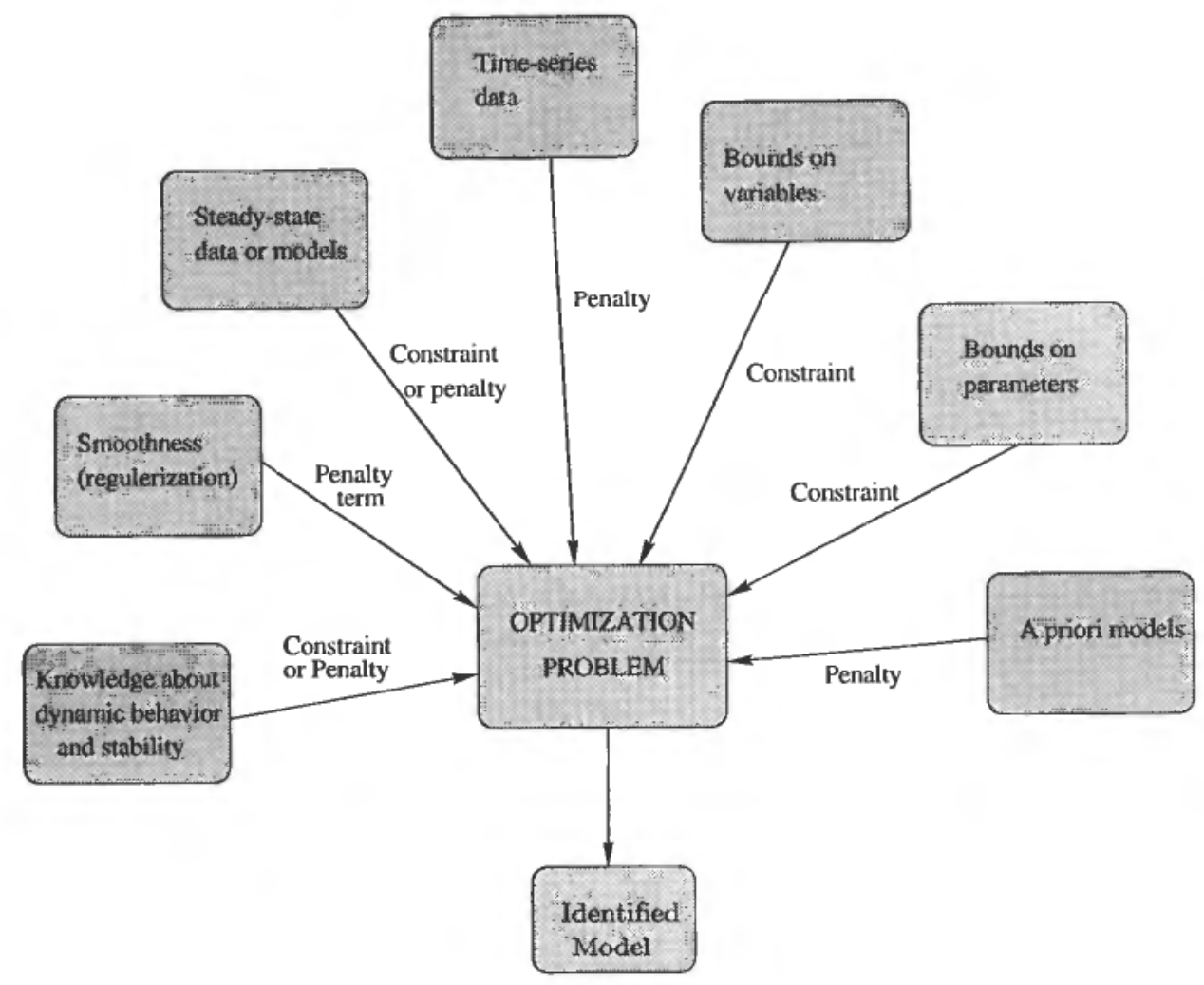

Figure 1. Prior knowledge in terms of penalties and constraints can be combined with empirical data in an optimization formulation of the identification problem.

framework (Peterka 1981, Tulleken 1993, Kárný et al. 1995). Relevant optimization methods are discussed in Kunisch and Sachs (1992).

The main idea is that explicit application of prior knowledge will improve the robustness of the identification algorithm, eventually leading to more accurate or useful parameter estimates, in some sense.

The continuation of this paper is as follows: In section 2, we formulate the identification problem with regularization and constraints as an optimization problem, taking the standard PEM as the starting point. The solution to this problem is briefly discussed. The main part of this paper, section 3, describes how the MSE (Mean Squared prediction Error) can be estimated. With the standard PEM, the FPE statistic is a well known estimate that is asymptotically unbiased under some conditions (Akaike 1969, Söderström and Stoica 1988). A generalization of the FPE statistic to cover regularized models was derived in Larsen and Hansen (1994). Here, this statistic is generalized further to also cover constrained and regularized system identification problems.

\section{Regularization and constraints}

Suppose a model structure, i.e. a set of equations parameterized by a $d$-dimensional parameter vector $\theta$, is given. The parameter vector can be estimated using a standard prediction error estimator, e.g. (Söderström and Stoica 1988) 


$$
\begin{gathered}
\hat{\theta}_{P E}\left(Z^{N}\right)=\arg \min _{\theta \in D_{c}} V_{N}\left(\theta ; Z^{N}\right) \\
V_{N}\left(\theta ; Z^{N}\right)=\frac{1}{N} \sum_{t=1}^{N} \varepsilon^{2}(t ; \theta)
\end{gathered}
$$

on the basis of a finite data sequence $Z^{N}=((u(1), y(1)),(u(2), y(2)), \ldots,(u(N), y(N)))$, where $\varepsilon(t ; \theta)=y(t)-\hat{y}\left(t ; Z^{t-1}, \theta\right)$, and $y(t)$ and $u(t)$ are the system's scalar outputs and inputs at time $t$, respectively. The one-step-ahead prediction $\hat{y}\left(t ; Z^{t-1}, \theta\right)$ is computed by solving the model equations with the parameter vector $\theta .{ }^{1}$ The parameter set $D_{c}$ is assumed to be compact, and the predictor is assumed to satisfy the necessary smoothness conditions such that a unique minimum of $V_{N}$ exists.

If the identifiability of the model is poor, or the data are not sufficiently informative, or the model structure is over-parameterized, or fundamentally wrong, the prediction error method may not be robust, giving highly uncertain estimates. In general, some form of prior knowledge can be applied to improve the robustness of the identification problem. An approach to introduce prior knowledge in terms of penalties and constraints to the prediction error method was described in Johansen (1996a), and briefly reviewed in the introduction. Such penalties are closely related to the method of regularization (Tikhonov and Arsenin 1977) as discussed in Johansen (1996a, 1997). Regularization is a general method for improving the robustness of mathematical algorithms by imposing additional regularity constraints on the solution. Mathematically, the problem we are now suggesting to solve has the form

$$
V_{N, \vartheta}^{\mathrm{REG}}\left(\theta ; Z^{N}\right)=V_{N}\left(\theta ; Z^{N}\right)+\gamma \Omega(\theta)
$$

subject to

$$
G(\theta)=0, K(\theta) \leq 0
$$

where $G$ and $K$ are smooth functions defining the equality and inequality constraints, $\Omega$ is a stabilizer for the problem, and $\gamma>0$ is a regularization parameter. ${ }^{2}$

The idea is that the penalty term $\Omega$ will attract any uncertain parameters in the model structure towards reasonable regions of the parameter space. Uncertain parameters are characterized by a low sensitivity of the criterion $V_{N}\left(\theta ; Z^{N}\right)$ with respect to perturbations in the corresponding sub-manifold of the parameter space. Hence, the penalty $\gamma \Omega(\theta)$ should contribute significantly to the criterion (relative to $V_{N}\left(\theta ; Z^{N}\right)$ ) when $\theta$ is in this sub-manifold. As mentioned in the introduction, the penalties can be selected on the basis of prior knowledge and desired properties such as e.g. smoothness, stability, convexity, additional equations not in the model, and explicit parameter knowledge.

The parameter estimate minimizing the above constrained and regularized prediction error criterion is denoted $\hat{\theta}_{\mathrm{REG}, \gamma}$. The asymptotic properties of this estimator in terms of bias and variance is discussed in detail in Johansen (1997) for the case when there are no constraints. A particular interesting property is that the total parameter error (bias plus variance) can be smaller than the Cramer-Rao lower bound, which is valid only for asymptotically unbiased estimates such as the PEM. It is expected that if the constraints are based on "correct prior knowledge", the asymptotic properties are

\footnotetext{
'The extension to multi-step-ahead predictors and systems with multiple inputs or outputs is straightforward.

${ }^{2}$ This formulation can be easily extended to the case with multiple penalties and regularization parameters.
} 
unchanged in the constrained case. If this assumption is violated, additional bias may be introduced, but in any case the variance is decreased.

The estimator properties when there are only constraints (no regularization) is discussed in some generality by Eskinat (1995).

\section{The final prediction error}

We define the MSE (Mean Squared prediction Error) as

$$
\mathrm{MSE}=E\left(y(t)-\hat{y}\left(t ; Z^{t-1}, \hat{\theta}\left(Z^{N}\right)\right)\right)^{2}
$$

for some arbitrary estimator $\hat{\theta}\left(Z^{N}\right)$. The variables $Z^{N}, Z^{t-1}$ and $y(t)$ are viewed as stochastic and $E$ is the expectation with respect to the joint distribution of these variables, so MSE is the ensemble average over all possible identification data sequences of length $N$, and future data sequences.

The MSE contains information about the expected prediction performance of the identified model. Such information is valuable for a number of purposes, including validation, selection of model structure and order, selection of the regularization parameter $\gamma$, and finding the optimal balance between bias and variance, see also (Xin et al. 1995). Unfortunately, since the underlying probability measures in system identification problems usually are unknown, the MSE must be estimated empirically. There exists a wide number of methods:

- A separate data sequence (independent of the identification data) can be used to estimate the MSE empirically. The major drawback is that an extra data sequence is needed. The other techniques we will discuss do not require this.

- The method of cross-validation is a widely applied technique (Stone 1974). The idea is to use a subsequence of the data for identification, and the remaining for estimating the MSE. This process is repeated for different subsequences, and the results are averaged. A closely related resampling technique is boot-strapping (e.g. Carlstein 1992). The major drawbacks of such resampling techniques are in general the additional computational complexity and the lack of strong theoretical results on their statistical properties.

- For the case without regularization and constraints, there are numerous closely related techniques such as the FPE (Final Prediction Error) statistic (Akaike 1969), the AIC (Akaike Information Criterion) (Akaike 1974), the MDL (Minimum Description Length) statistic (Rissanen 1978), the GCV (Generalized Cross-Validation) statistic (Craven and Wahba 1979) and other approximative cross-validation statistics (Stoica et al. 1986). They all make use of the average residuals, but make a correction for the dependence between the residuals using asymptotic considerations.

The main purpose of this paper is to discuss the extension of the classical FPE statistic

$$
\operatorname{FPE}\left(Z^{N}\right)=\frac{1+d / N}{1-d / N} V_{N}\left(\hat{\theta}_{P E} ; Z^{N}\right)
$$

derived from the standard prediction error method formulation to the generalized formulation with regularization and constraints. 


\subsection{FPE with Constraints}

First, we observe that the introduction of constraints (but no regularization) leads to a trivial modification of the FEP:

$$
\operatorname{FPEC}\left(Z^{N}\right)=\frac{1+d_{0} / N}{1-d_{0} / N} V_{N}\left(\hat{\theta}_{P E} ; Z^{N}\right)
$$

where the degrees of freedom is $d_{0}=d-d_{a}$. Here $d_{a}$ is the number of linearly independent and active linearized constraints at the point $\hat{\theta}_{P E}$. This modification can be made rigorous by the application of the Implicit Function Theorem in order to reparameterize the criterion function at the point $\hat{\theta}_{P E}$ in terms of a $d_{0}$ dimensional parameter vector, as suggested in Johansen (1996a).

\subsection{FPE with Regularization}

Second, an extension of the FPE to the case when there is regularization (but no constraints) can be found in Larsen and Hansen (1994):

$$
\operatorname{FPER}\left(\gamma ; Z^{N}\right)=\frac{1+d_{2}(\gamma) / N}{1-2 d_{2}(\gamma) / N+d_{1}(\gamma) / N} \cdot V_{N}\left(\hat{\theta}_{\mathrm{REG}, \gamma} ; Z^{N}\right)
$$

where the two different expressions for the model's degrees of freedom are given by $d_{1}(\gamma)=\operatorname{tr}(S(\gamma))$ and $d_{2}(\gamma)=\operatorname{tr}(S(\gamma) S(\gamma))$, where

$$
\begin{aligned}
S(\gamma) & =\left(H_{N}\left(\hat{\theta}_{\mathrm{REG}, \gamma}\right)+\gamma H_{\Omega}\left(\hat{\theta}_{\mathrm{REG}, \gamma}\right)\right)^{-1} \cdot H_{N}\left(\hat{\theta}_{\mathrm{REG}, \gamma}\right) \\
H_{N}(\theta) & =\nabla_{\theta}^{2} V_{N}\left(\theta ; Z^{N}\right) \\
H_{\Omega}(\theta) & =\nabla_{\theta}^{2} \Omega(\theta)
\end{aligned}
$$

\subsection{FPE with Constraints and Regularization}

Finally, we discuss the case when both regularization and constraints are being applied. The idea is to reparameterize the FPER statistic in terms of a lower-dimensional parameter vector by eliminating parameters corresponding to the active constraints, and compute the value of the reparameterized FPER criterion. This was suggested in Johansen (1996a), and the details follow. tions:

In a neighborhood of $\hat{\theta}_{\mathrm{REG}, \gamma}$, the constraints are approximation by their lineariza-

$$
A_{G} \theta-b_{G}=0, \quad A_{K} \theta-b_{K} \leq 0
$$

where

$$
\begin{aligned}
A_{G} & =\nabla_{\theta} G\left(\hat{\theta}_{\mathrm{REG}, \gamma}\right) \\
A_{K} & =\nabla_{\theta} K\left(\hat{\theta}_{\mathrm{REG}, \gamma}\right) \\
b_{G} & =\nabla_{\theta} G\left(\hat{\theta}_{\mathrm{REG},_{\gamma}, \gamma} \hat{\theta}_{\mathrm{REG}, \gamma_{\gamma}}-G\left(\hat{\theta}_{\mathrm{REG}, \gamma}\right)\right. \\
b_{K} & =\nabla_{\theta} K\left(\hat{\theta}_{\mathrm{REG}, \gamma}\right) \hat{\theta}_{\mathrm{REG}, \gamma}-K\left(\hat{\theta}_{\mathrm{REG}, \gamma}\right)
\end{aligned}
$$

Keeping only the active and linearly independent constraints at $\hat{\theta}_{\mathrm{REG}, \gamma}$, it is clear that $\hat{\theta}_{\mathrm{REG}, \gamma}$ satisfies: 


$$
A \hat{\theta}_{\mathrm{REG}, \gamma}-b=0
$$

where $\operatorname{dim}(A)=d_{a} \times d_{a}$, and $A$ contains the active and linearly independent rows of $A_{G}$ and $A_{K}$. Likewise, $b$ contains the corresponding elements of $b_{G}$ and $b_{K}$. Without loss of generality, ${ }^{3}$ it can be assumed that the $d$-dimensional vector $\theta$ can be decomposed into a pair of $d_{a}$ and $\left(d-d_{a}\right)$-dimensional sub-vectors $\theta_{1}$ and $\theta_{2}$ and the $A$ matrix into two sub-matrices $A_{1}$ and $A_{2}$ such that

$$
\left(\begin{array}{ll}
A_{1} & A_{2}
\end{array}\right)\left(\begin{array}{l}
\theta_{1} \\
\theta_{2}
\end{array}\right)=b
$$

$\operatorname{dim}\left(A_{1}\right)=d_{a} \times d_{a}, \operatorname{dim}\left(A_{2}\right)=d_{a} \times\left(d-d_{a}\right)$, and $\operatorname{rank}\left(A_{1}\right)=d_{a}$. Now, it is clear that $\theta_{1}$ satisfies

$$
\theta_{1}=A_{1}^{-1}\left(b-A_{2} \theta_{2}\right)
$$

Hence, an approximate (linearized) reparameterization of $\theta$ in terms of the $\left(d-d_{a}\right)$-dimensional vector $\theta_{2}$ is defined by

$$
\theta=\left(\begin{array}{c}
\theta_{1} \\
\theta_{2}
\end{array}\right)=\left(\begin{array}{c}
A_{1}^{-1}\left(b-A_{2} \theta_{2}\right) \\
\theta_{2}
\end{array}\right)=P \theta_{2}-q
$$

where the least inequality is an implicit definition of $P$ and $q$. Notice that the above approximation is exact at the point $\hat{\theta}_{\mathrm{REG}, \gamma}$. Defining

$$
\hat{\theta}_{\mathrm{REG}, \gamma}^{T}=\left(\hat{\theta}_{1, \mathrm{REG}, \gamma}^{T}, \hat{\theta}_{2, \mathrm{REG}, \gamma}^{T}\right)
$$

it is clear that

$$
\begin{aligned}
V_{N}\left(\hat{\theta}_{\mathrm{REG}, \gamma} ; Z^{N}\right) & =V_{N}\left(P \hat{\theta}_{2, \mathrm{REG}, \gamma}-q ; Z^{N}\right) \\
& =\tilde{V}_{N}\left(\hat{\theta}_{2, \mathrm{REG}, \gamma}\right)
\end{aligned}
$$

where the last inequality is a definition of the reparameterized criterion $\tilde{V}_{N}$. Now we are in a position to apply the FPER statistic to define FPECR since the constraints are removed in the re-parameterized version of the criterion:

$$
\operatorname{FPECR}\left(\gamma ; Z^{N}\right)=\frac{1+\tilde{d}_{2}(\gamma) / N}{1-2 \tilde{d}_{2}(\gamma) / N+\tilde{d}_{1}(\gamma) / N} \cdot V_{N}\left(\hat{\theta}_{\mathrm{REG}, \gamma} ; Z^{N}\right)
$$

where the two different expressions for the model's degrees of freedom are given by $\tilde{d}_{1}(\gamma)=\operatorname{tr}(\tilde{S}(\gamma))$ and $\tilde{d}_{2}(\gamma)=\operatorname{tr}(\tilde{S}(\gamma) \tilde{S}(\gamma))$. Moreover,

$$
\tilde{S}(\gamma)=\left(\tilde{H}_{N}\left(\hat{\theta}_{2, \mathrm{REG}, \gamma}\right)+\gamma \tilde{H}_{\Omega}\left(\hat{\theta}_{2, \mathrm{REG}, \gamma}\right)\right)^{-1} \cdot \tilde{H}_{N}\left(\theta_{2, \mathrm{REG}, \gamma}\right)
$$

where

$$
\begin{aligned}
& \tilde{H}_{N}\left(\theta_{2}\right)=\nabla_{\theta_{2}}^{2} \tilde{V}_{N}\left(\theta_{2} ; Z^{N}\right) \\
& \tilde{H}_{\Omega}\left(\theta_{2}\right)=\nabla_{\theta_{2}}^{2} \Omega\left(\theta_{2}\right)
\end{aligned}
$$

\footnotetext{
${ }^{3}$ The ordering of the elements in the vector $\theta$ can be rearranged to achieve this.
} 


\subsection{Discussion}

The derivation of the classical Final Prediction Error is based on first order Taylor-expansions (linearization) (Söderström and Stoica 1988). The error introduced by the linearization of the constraints is therefore expected to be of the same order as the other approximation error in the derivations. The asymptotic statistical properties of the FPECR criterion is therefore expected to be similar to those of the FPER criterion, see Larsen and Hansen (1994).

The above criteria can be easily generalized to a MIMO system identification framework, including multiple regularization penalties with separate regularization parameters.

Some of the above methods for regularization and FPE-like statistics are implemented as part of a computer-aided modeling tool for developing operating regime based models (Johansen 1996b, Johansen and Foss 1997), which are closely related to Takagi-Sugeno-Kang fuzzy models (Takagi and Sugeno 1985).

\section{Concluding remarks}

A general framework for introducing prior knowledge in terms of penalties (including regularization) and constraints into non-linear system identification problems was described in Johansen (1996a). In the present paper, the FPE statistic is extended to handle the case when the prediction error criterion is augmented with penalties and constraints, leading to the FPECR. This statistic is useful both for model structure identification and selection of regularization parameters.

\section{REFERENCES}

AKAIKE, H. (1969). Fitting autoregressive models for prediction. Ann. Inst. Stat. Math. 21, 243-247.

AKAIKE, H. (1974). A new look at the statistical model identification. IEEE Trans. Automatic Control 19, 716-723.

BAI, E. W. and SASTRY, S. S. (1986). Parameter identification using prior information. Int. J. Control 44, 455-473.

CARlsteIn, E. (1992). Resampling techniques for stationary time-series: Some recent developments. In: New Direction in Time Series Analysis, Part I (D. Brillinger et al., Ed.), pp. 75-85. Springer-Verlag, New York, NY.

CRAVEN, P. and WAHBA, G. (1979). Smoothing noisy data with spline functions. Estimating the correct degree of smoothing by the method of generalized cross-validation. Numerical Math. 31, 317-403.

DAYAL, B. S. and MACGREGOR, J. F. (1996). Identification of finite impulse response models: Methods and robustness issues. Industrial and Engineering Chemistry Research 35, 4078-4090.

De Moor, B., Gevers, M. and Goodwin, G. C. (1994). $l_{2}$-overbiased, $l_{2}$-underbiased and $l_{2}$-unbiased estimation of transfer functions. Automatica 30, 893-898.

Eskinat, E. (1995). System identification using constrained estimation. In: Proc. European Control Conference, Rome, pp. 856-861.

EsKINAT, E., JoHNSON, S. and LUYBEN, W. (1993). Use of auxiliary information in system identification. Ind. Eng. Chem. Research 32, 1981-1992.

Foss, B. A. and JOHANSEN, T. A. (1997). Identification and convexity in optimizing control. Preprints IFAC Symposium on System Identification, Kitakyushu, Japan, pp. 691-696.

Gawthrop, P. J., Jones, R. W. and MACKENZIE, S. A. (1992). Identification of partially-known systems. Automatica 28, 831-836.

JOHANSEN, T. A. (1996a). Identification of non-linear systems using empirical data and prior knowledge-An optimization approach. Automatica 32, 337-356.

JOHANSEN, T. A. (1996b). Robust identification of Takagi-Sugeno-Kang fuzzy models using regularization. In: Proc. IEEE Conf. Fuzzy Systems, New Orleans, pp. 180-186. 
JOHANSEN, T. A. (1997). On Tikhonov regularization, bias and variance in nonlinear system identification. Automatica 33, 441-446.

JOHANSEN, T. A. and Foss, B. A. (1997). ORBIT-operating regime based modeling and identification toolkit. Preprints IFAC Symposium on System Identification, Kitakyushu, Japan, pp. 961-968.

KÁRNÝ, M., HAlOUSKOVÁ, A. and NedOMA, P. (1995). Recursive approximation by ARX model: A tool for grey-box modelling. Int. J. Adaptive Control and Signal Processing 9, 525-546.

Kramer, M. A., ThOmPSON, M. L. and PHAGAT, P. M. (1992). Embedding theoretical models in neural networks. In: Proceedings American Control Conference, Chicago, Il., pp. 475-479.

KUNISCH, K. and SACHS, E. W. (1992). Reduced SQP methods for parameter identification problems. SIAM J. Numerical Analysis 29, 1793-1820.

LARSEN, J. and HANSEN, L. K. (1994). Generalization performance of regularized neural network models. In: Proc. IEEE Workshop on Neural Networks for Signal Processing, Ermioni, Greece.

Moons, C. and DE MOOR, B. (1995). Parameter identification of induction motor drives. Automatica 31, 1137-1147.

PETERKA, V. (1981). Bayesian system identification. Automatica 17, 41-53.

RisSANEN, J. (1978). Modeling by shortest data description. Automatica 14, 465-471.

SJÖBERG, J., HJALmARSSON, H. and LJUNG, L. (1994). Neural networks in system identification. In: Preprints 10th IFAC Symp. System Identification, Copenhagen. Vol. 2, pp. 49-72.

SuöBerg, J., McKelvey, T. and LJung, L. (1993). On the use of regularization in system identification. In: Preprints 12th IFAC World Congress, Sydney. Vol. 7, pp. 381-386.

SÖDERSTRÖM, T. and STOICA, P. (1988). System Identification. Prentice Hall, Englewood Cliffs, NJ.

Stoica, P., EYKhOFf, P., JANSSEN, P. and SöDERSTRÖM, T. (1986). Model-structure selection by cross-validation. Int. J. Control 43, 1841-1878.

STONE, M. (1974). Cross-validatory choice and assessment of statistical predictions. J. Royal Statistical Soc. B 36, 111-133.

TAKAGI, T. and SugENO, M. (1985). Fuzzy identification of systems and its application to modeling and control. IEEE Trans. Systems, Man, and Cybernetics 15, 116-132.

Thompson, M. L. and Kramer, M. A. (1994). Modeling chemical processes using prior knowledge and neural networks. AIChE J. 40, 1328-1340.

TikHonov, A. N. and ARSEnIN, V. Y. (1977). Solutions of Ill-posed Problems. Winston, Washington DC.

TULLEKEN, H. J. A. F. (1993). Grey-box modelling and identification using physical knowledge and Bayesian techniques. Automatica 29, 285-308.

XIN, J., OHMORI, H. and SANO, A. (1995). Minimum MSE based regularization for system identification in the presence of input and output noise. In: Proc. 34th IEEE Conf. Decision and Control, New Orleans, pp. 1807-1814. 